\title{
PREVALÊNCIA DE SUPLEMENTAÇÃO DE VITAMINA B12 EM VEGETARIANOS DA UNIVERSIDADE REGIONAL DE BLUMENAU (FURB)
}

\author{
THE PREVALENCE OF SUPPLEMENTATION OF B12 VITAMIN IN \\ VEGETARIAN OF BLUMENAU REGIONAL UNIVERSITY (BRU)
}

\author{
Rudolf, $\mathrm{PA}^{1}$, Cerutti, $\mathrm{LM}^{2}$, Cordova, $\mathrm{MM}^{3}$, Salla, L ${ }^{4}$, Valente, $\mathrm{C}^{5}$ \\ Afiliações: 1- Departamento de Biomedicina da Universidade Regional de Blumenau- FURB. 2- Faculdade de Medicina da Universidade \\ Regional de Blumenau- FURB. 3 - Departamento de Ciências Naturais da Universidade Regional de Blumenau - FURB. 4- Centro de \\ Ciências Aplicadas à Saúde da Universidade Estadual do Oeste do Paraná - UNIOESTE. 5- Departamento de Ciências Naturais da \\ Universidade Regional de Blumenau - FURB. \\ Misael Siqueira Bello/133, Nossa Senhora de Fátima, Barracão, Paraná, Brasil. CEP: 85700-000. larissasalla@ hotmail.com.br
}

\section{Resumo}

A dieta vegetariana está se tornando mais comum no mundo. No Brasil cerca de $14 \%$ da população é vegetariana, representando cerca de 30 milhões de pessoas, segundo a Sociedade Vegetariana Brasileira. O objetivo do trabalho é avaliar a prevalência da suplementação de vitamina B12 em vegetarianos da Universidade Regional de Blumenau (FURB). A vitamina B12 é sintetizada por bactérias e encontrada em produtos de origem animal, não sendo encontrada em quantidades adequadas em produtos de origem vegetal, pois as plantas não necessitam de vitamina B12. A pesquisa foi realizada com alunos vegetarianos. Foi aplicado um questionário com 13 perguntas, disponibilizado por formulário Google e enviado por e-mail, para 20 pessoas, com perguntas a respeito de suplementação de vitaminas B12 e vegetarianismo. Os resultados foram transformados em gráficos para melhor compreensão e interpretação dos dados apurados. Constatou-se que $60 \%$ dos participantes eram ovo-lacto-vegetarianos e $40 \%$ vegetarianos estritos, $50 \%$ fazem suplementação de vitamina B12 onde a maioria faz suplementação oral sendo que $15 \%$ utilizam 2,4 a $100 \mathrm{mcg}$ por dia. É possível observar que $\mathbf{7 5 \%}$ dos participantes da pesquisa fazem acompanhamento com exames bioquímicos, o que é importante para avaliar a necessidade de suplementação. Em relação aos sintomas, $35 \%$ não apresentam sintomas, fato este que pode estar associado ao curto período de dieta, menos de 3 anos. Já que a vitamina B12 possui estoque hepático. Concluiu-se que a suplementação de vitamina B12, mesmo que necessária não é amplamente adotada pelos vegetarianos analisados neste estudo, sendo suplementada por metade dos participantes.

Palavras-chave: Vitamina; Vitamina B12; Vegetariano; Suplementação; FURB

\section{Abstract}

The vegetarian diet is common in the world. In Brazil about $14 \%$ of the population is vegetarians, representing around 30 million people, according to estimates of the Brazilian Vegetarian Society. The objective of this study is to evaluate the prevalence of vitamin B12 supplementation in vegetarians at the Regional University of Blumenau (FURB), since vitamin B12 is synthesized by bacteria and found in animal products, isn't present in adequate quantities in products of plant origin, since the plants don't need vitamin B12. The research was carried out with vegetarians FURB students. A questionnaire with 13 questions was applied by Google form and sent by e-mail, for 20 people, with questions regarding vitamin $\mathrm{B} 12$ supplementation and vegetarianism. The results were transformed into graphs to better understand and interpret the data. It was found that $60 \%$ of the participants were lacto-ovo-vegetarians and $40 \%$ strict vegetarians, 50\% made vitamin B12 supplementation where the majority does oral supplementation and $15 \%$ use 2.4 to $100 \mathrm{mcg}$ per day. It is possible to observe that $75 \%$ of the participants of the research are followed up with biochemical tests, which is important to evaluate the need for supplementation. Regarding the symptoms, $35 \%$ do not present symptoms, fact that can be associated to the short period of diet, less than 3 years. Since vitamin B12 has hepatic stock. It was concluded that vitamin B12 supplementation, although necessary, is not adopted by the vegetarians in this study, even most doing supplementation.

Keywords: Vitamin; B12 vitamin; Vegetarian; Supplementation; FURB. 


\section{Introdução}

A prática da dieta vegetariana vem aumentando nos últimos anos no mundo. Estima-se que no Brasil, cerca $14 \%$ da população de brasileiros se declaram vegetarianos, representando cerca de 30 milhões de pessoas, segundo estimativas da Sociedade Vegetariana Brasileira (SVB) baseada em dados do Instituto de Opinião Pública e Estatística (IBOPE), com um aumento de $75 \%$ em relação à última pesquisa em $2012^{8}$.

Os motivos são multifatoriais dentre eles destacam-se as questões de maus tratos a animais, saúde e questões ambientais. Hoje se sabe que a dieta vegetariana, se bem planejada, possui inúmeros benefícios como diminuição do risco de obesidade, doenças cardiovasculares e diabetes $^{3}$.

Segundo estudos, dentre todos os nutrientes ingeridos pelas populações vegetarianas, apenas a vi4amina B12 pode estar inadequada, o que mostra a importância da suplementação da vitamina B $12^{5}$.

A vitamina B12 é produzida por bactérias, e encontrada em produtos de origem animal. As plantas não possuem vitamina B12, já que não necessitam da mesma para seu funcionamento ${ }^{6}$.

As recomendações diárias de ingestão de vitamina B12 para seres humanos após os 14 anos de idade (fora do período de gestação ou lactação) são 2,4 mcg/dia, sendo a necessidade de absorção de $1 \mathrm{mcg} / \mathrm{dia}$, e a concentração segura de vitamina B12 no sangue, deve ficar sempre acima de $350 \mathrm{pg} / \mathrm{mL}$, para garantir mais segurança a concentração deve ficar acima de $490 \mathrm{pg} / \mathrm{mL}^{6}$.

Diversos estudos demonstram que grupos vegetarianos apresentam menor ingestão de vitamina B12, e têm menores concentrações séricas. No entanto, as concentrações séricas de onívoros, apesar de maior do que dos vegetarianos, também são inadequados para a boa manutenção da saúde ${ }^{1}$. O vegetariano estrito deve fazer a suplementação para garantir uma dieta saudável ${ }^{4}$. Sabendo da importância do consumo adequado da Vitamina B12 para o ser humano e prevalências de sua carência em vegetarianos estritos, este trabalho tem por objetivo avaliar a prevalência da suplementação de vitamina B12 neste grupo de indivíduos. 


\section{Métodos}

O trabalho foi realizado na FURB Universidade Regional de Blumenau com alunos de qualquer curso com a obrigatoriedade de serem vegetarianos. A sua condução foi aprovada pelo Comitê de Ética em Pesquisa em Seres Humanos, sob o parecer $n^{\circ}$ 2.908.064. Todos os participantes leram e assinaram o Termo de Consentimento Livre e Esclarecido e pesquisa. quantitativo, tendo em vista uma breve revisão bibliográfica para compreensão sobre a importância da vitamina B12. Foi aplicado um questionário (apêndice) aos alunos da FURB tendo este por objetivo avaliar a prevalência de suplementação da vitamina B12 em indivíduos vegetarianos da FURB.

O estudo foi realizado com 20 pessoas, por meio de um questionário através do ambiente virtual, que foi disponibilizado por e-mail. Os participantes que se disponibilizaram foram orientados sobre o objetivo e os procedimentos do estudo, com a obrigatoriedade de serem vegetarianos, estudem na FURB e maiores de 18 anos, foram excluídos aqueles que não se estavam cientes de como iria funcionará a

Nesse estudo foi utilizado o método

disponibilizaram a responder o questionário ou que não respondessem alguma questão.

$\mathrm{O}$ instrumento que foi utilizado para a coleta de dados constituiu-se em um questionário estruturado que contém 13 perguntas relacionadas aos hábitos e costumes do vegetarianismo.

O questionário foi respondido via internet (e-mail), pelo site de Formulários Google e abordou primeiramente perguntas pessoais, como sexo, idade, depois perguntas relacionadas ao vegetarianismo, como por exemplo: Como você se classifica no vegetarianismo? E Quanto tempo você é vegetariano? Seguido de perguntas sobre a suplementação da vitamina B12.

Os dados foram analisados por meio das frequências absolutas e médias que foram obtidos pelo site Formulários Google. As informações obtidas através do questionário foram apresentadas através de gráficos para a melhor compreensão e interpretação dos dados apurados.

\section{Resultados}


A figura 1 apresenta o sexo e idade dos participantes. Como apontado na figura 1A, $90 \%$ $(n=18)$ dos participantes são do sexo feminino e $10 \%(\mathrm{n}=2)$ são do sexo masculino. Na figura 1B apontando a idade de 18 a 20 anos $20 \%(\mathrm{n}=4)$, 21 a 25 anos $55 \%(\mathrm{n}=11), 26$ a 30 anos $15 \%$ $(n=3)$ e 31 a 36 anos $10 \%(n=2)$.
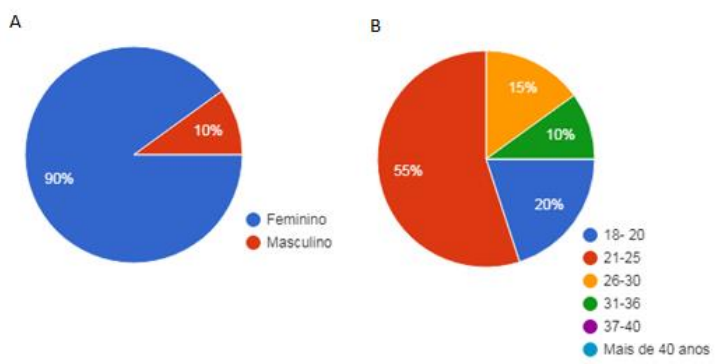

Figura 1. Sexo e idade dos participantes Fonte: Autor (2018)

A figura 2 apresenta a classificação do vegetarianismo (A) e quanto tempo o participante é vegetariano (B).

$\mathrm{Na}$ figura $2 \mathrm{~A}$ podemos perceber que $60 \%(n=12)$ dos participantes se classificam como Ovo-lacto-vegetarianos, $25 \% \quad(\mathrm{n}=5)$ veganos e $15 \%(n=3)$ como vegetarianos estritos. $\mathrm{Na}$ figura 2B mostra quanto tempo o participante é vegetariano. Sendo $50 \%(\mathrm{n}=10)$ de 1 a 5 anos, $30 \%(n=6)$ de 6 meses a 1 ano, $15 \%(n=3)$ de 6 a 10 anos e $10 \%(n=1)$ mais de 10 anos.
A figura 3 representa a presença de suplementação e quanto tempo o participante está suplementando.
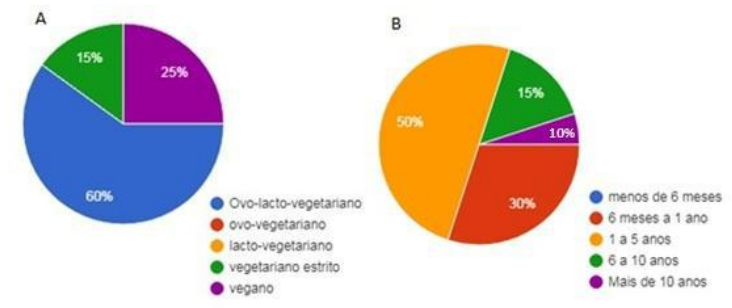

Figura 2. Classificação do vegetarianismo e tempo de duração da dieta

Fonte: Autor (2018)

Na figura $2 A$ vemos que $50 \%(n=10)$ dos participantes fazem suplementação de vitamina B12 e os outros 50\% (n=10) não suplementam.

Já na figura 3B temos a informação quanto ao tempo de suplementação, sendo que $20 \%(n=4)$ dos participantes que suplementam, fazem a suplementação de 1 a 5 anos, 20\% (n=4) fazem a menos de 6 meses, $10 \%(\mathrm{n}=1) 6$ meses a 1 ano, $10 \%(n=1) 2$ a 5 anos e $50 \%(n=10)$ não fazem a suplementação de vitamina B12. Pelo fato da maioria das pessoas, $50 \%(\mathrm{n}=10)$, terem adotado esse novo estilo de vida há pouco tempo (de 1 a 5 anos), e outros $30 \%$ (n=6) (6 meses a 1 ano), pode existir uma correlação entre o desconhecimento da importância da 
suplementação da vitamina B12 e a falta da mesma.
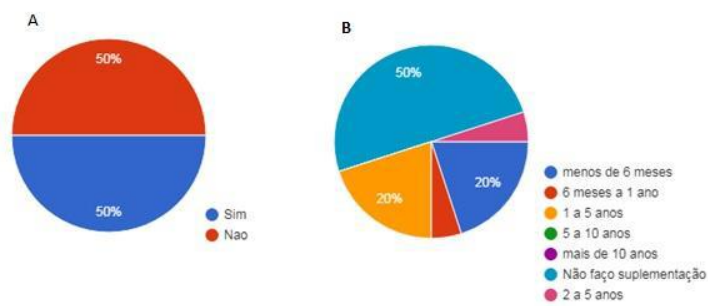

Figura 3. Presença de suplementação e tempo de suplementação Fonte: Autor (2018)

A figura 4 representa qual frequência de suplementação e qual o meio utilizado. Na figura 4A podemos observar $30 \%(n=6)$ fazem a suplementação diariamente, $10 \% \quad(\mathrm{n}=2)$ anualmente, $5 \%(n=1)$ semanalmente e $5 \%(n=1)$ mensalmente. Metade dos vegetarianos que participaram da pesquisa fazem o uso de suplementação de vitamina B12, cerca de $50 \%$ $(n=10)$.
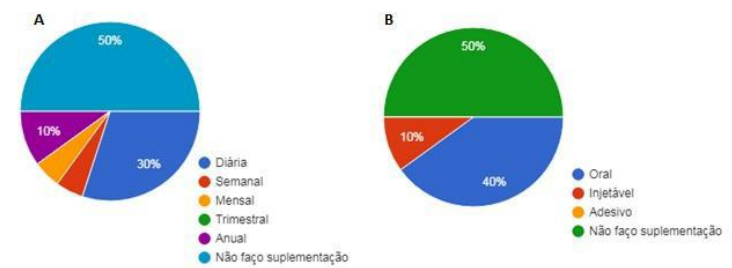

Figura 4. Frequência e meio de suplementação. Fonte: Autor (2018).

$\mathrm{Na}$ figura $4 \mathrm{~B}$ vemos qual o meio de suplementação utilizado, onde $40 \%(\mathrm{n}=8)$ dos participantes utilizam a forma oral e $10 \%(n=2)$ a forma injetável.

A figura 5 representa qual a dosagem de vitamina B12 utilizada. Vemos que $15 \%(n=3)$ utilizam de 2,4 a $100 \mathrm{mcg}, 10 \%(\mathrm{n}=2)$ de $200 \mathrm{a}$ $50 \mathrm{mcg}, 10 \%(\mathrm{n}=2)$ de 600 a $1000 \mathrm{mcg}, 10 \%$ $(n=2) 5000 \mathrm{mcg}$ e $5 \%(n=1)$ de 1500 a $3000 \mathrm{mcg}$. Devido à algumas características exclusivas do metabolismo da B12, doses de até $1.000 \mathrm{mcg}$ por dia podem ser prescritas pelo nutricionista de acordo com a Anvisa e o Conselho Federal de Nutrição ${ }^{7}$.

A figura 6 representa a presença de exames bioquímicos para verificar as concentrações de vitamina B12 e qual os exames são utilizados.

$\mathrm{Na}$ figura $6 \mathrm{~A}$ vemos $25 \%(\mathrm{n}=5)$ dos participantes não fazem exames bioquímicos para vitamina $\mathrm{B} 12, \quad 45 \% \quad(\mathrm{n}=9) \quad$ fazem anualmente, $20 \%(n=4)$ fazem semestralmente e $10 \%(\mathrm{n}=2)$ fazem trimestralmente. 


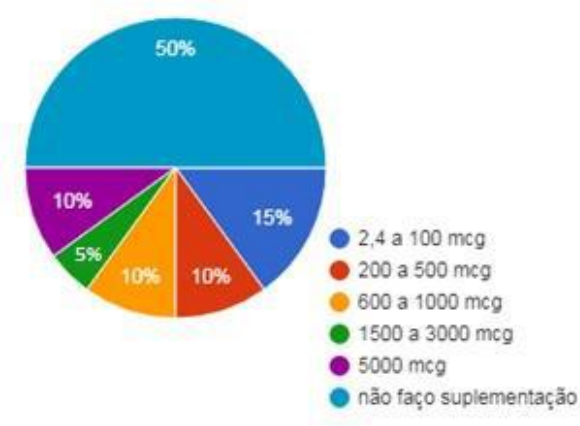

Figura 5. Dosagem de vitamina B12 utilizada. Fonte: Autor (2018).

Na figura 6 B vemos quais os tipos de exames são mais utilizados pelos participantes. Onde $57,1 \%(n=12)$ dos participantes fazem dosagem de vitamina $\mathrm{B} 12,9,5 \% \quad(\mathrm{n}=2)$ fazem dosagem de homocisteína, 9,5\% (n=2) dosagem de ácido metilmalônico e 23,8\% não fazem exames bioquímicos para vitamina B12.
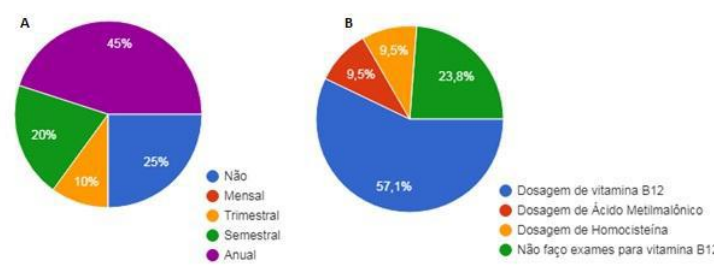

Figura 6. Presença de exames bioquímicos. Fonte: Autor (2018).

A figura 7 representa os sintomas que podem estar relacionados à deficiência de vitamina B12, pois são sintomas gerais, podendo ocorrer em diversas patologias. Podemos ver que $35 \%(\mathrm{n}=7)$ dos participantes não possuem sintomas, $25 \%(\mathrm{n}=5)$ possuem a exaustão, e em geral os participantes possuem algum outro tipo de sintoma, como nervosismo, palidez, confusão mental, depressão e formigamento (pés e mãos).

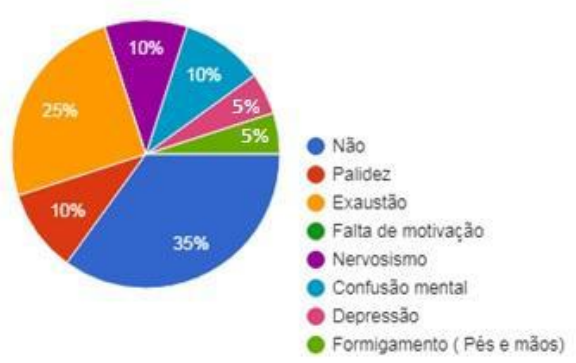

Figura 7. Sintomas relacionados à deficiência de vitamina B12. Fonte: Autor (2018).

A figura 8 representa o conhecimento dos vegetarianos sobre a importância da suplementação da vitamina B12. Onde vemos que $65 \%(n=13)$ dos participantes acham que os vegetarianos estão bem informados sobre a importância da suplementação de vitamina B12 e 35\% (n=7) acham que não população não está bem informada.

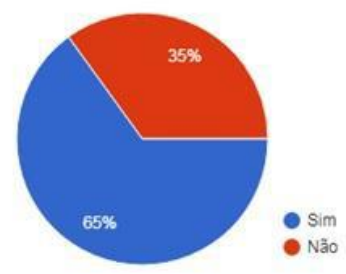

Figura 8. Conhecimento sobre B12. Fonte: Autor (2018). 


\section{Discussão}

A dieta ovo-lacto-vegetariana pode oferecer a vitamina B12 necessária ao organismo, desde que haja um consumo regular de ovos e laticínios, tudo depende do metabolismo de cada indivíduo. Já a dieta vegetariana estrita sem a presença de alimentos fortificados com vitamina B12 deve ser suplementada. Esse é o único nutriente que pode estar ausente na dieta vegetariana estrita ${ }^{7}$.

A suplementação de vitamina B12 pode ser feita em forma de cápsulas vegetais ou gotas, na dose de pelo menos $10 \mathrm{mcg}$ por dia, para que se tenha uma concentração adequada ${ }^{6}$.

Em um estudo conduzido ${ }^{2}$, constatou-se que $10,8 \%$ dos ovo-lacto-vegetarianos e $50 \%$ dos vegetarianos estritos faziam uso de suplementação apenas de vitamina B12. Porém, $50 \%(n=10)$ dos participantes não fazem a suplementação da vitamina, estando relacionada com aversão de alimentos artificiais, por parte dos veganos.

\section{Conclusão}

A suplementação de vitamina B12, mesmo sendo necessária não é amplamente adotada pelos vegetarianos analisados neste estudo, sendo suplementada apenas por metade dos participantes. Os ovo-lacto-vegetarianos podem obter quantidades significativas dessa vitamina, quando o consumo de ovos e laticínios é regular, já os vegetarianos estritos não obtêm vitamina B12 sem ser através de alimentos fortificados ou pela suplementação.

Sabe-se que essa vitamina tem um estoque no fígado de 3 a 5 anos, mas não é possível determinar quanto tempo durará, devido a diversos fatores que influenciam o seu consumo metabólico, como atividade neuronal, ciclo entero-hepático, metabolismo hepático, uso de medicamentos etc. Sendo assim, todo vegetariano (estrito ou ovo-lacto-vegetariano), independentemente do tempo de dieta, devem avaliar as concentrações dessa vitamina e se necessário suplementa-la.

Sabe-se que a deficiência de vitamina B12 acomete de $50 \%$ a $60 \%$ dos vegetarianos, na dieta onívora cerca de $50 \%$ dos indivíduos também possuem deficiência dessa vitamina, o que nos mostra a importância dessa vitamina, pouco conhecida.

É importante saber que a vitamina B12 dos suplementos não é de origem animal, sendo obtida por cultivo de bactérias em laboratório, 
não interferindo na ideologia da dieta vegetariana. É necessário que seja feita a suplementação da vitamina B12 em indivíduos que adotaram esse estilo de vida, pois a deficiência desta pode causar problemas neurológicos, hematológicos e cardiovasculares. Os sintomas muitas vezes não são aparentes e por isso não é dado o devido valor a essa vitamina, mas quando os sintomas começam a aparecer pode ser um indício que a deficiência já está muito avançada, podendo o tratamento não ser reversível.

Esta pesquisa pode contribuir para um maior conhecimento da suplementação da vitamina $\mathrm{B} 12$, em vegetarianos, pode colaborar com subsídios para possíveis intervenções futuras, orientado de forma assertiva este grupo sobre a importância da inclusão do nutriente na dieta diária.

O exame bioquímico da vitamina B12 mostra a concentração da vitamina reduzida no sangue e juntamente com isso a concentração elevada de homocisteína e de ácido metilmalônico 5 . Sendo essa vitamina, essencial para a manutenção da bioquímica celular e em diversas reações orgânicas específicas, demonstrando a importância do acompanhamento das concentrações séricas destes pacientes ${ }^{2}$ Diante disto, o acompanhamento deste grupo de indivíduos fazse necessário para a garantida do seu estado nutricional, podendo ser necessário a suplementação deste nutriente.

A deficiência de vitamina B12 está associada à diminuição na atividade da mutase L-metilmonalil CoA que consequentemente aumenta as concentrações de ácido metilmalônico no sangue 9 . Uma vez que $25 \%$ $(\mathrm{n}=5)$ apresentaram o sintoma de exaustão, 5\% $(\mathrm{n}=1)$ relataram falta de motivação, $10 \%(\mathrm{n}=2)$ têm confusão mental, seguidos por nervosismo $10 \%(n=2)$ e depressão $5 \%(n=1)$.

\section{Referências}

1-KRAJCOVICOVA-KUDLACKOVA $\mathrm{M}$, et al. Traditional and alternative nutrition--levels of homocysteine and lipid parameters in adults. Scand J Clin Lab Invest. Pubmed [Internet]. 2000 Dec [cited 2018 Jun 15]; 60(8):657-64. Avaliable from: https://www.ncbi.nlm.nih.gov/pubmed/1121814 8 doi: 10.1080/00365510050216385

2-MIRANDA D, et al. Qualidade nutricional de dietas e estado nutricional de vegetarianos. Demetra Alimentação, Nutrição e Saúde. Epublicacoes [Internet]. 2013 [cite 2018 Jun 16]; 8(2):163-172. Avaliabe from:

https://www.e-

publicacoes.uerj.br/index.php/demetra/article/vi ew/4773/5167 
3-MORALEJO SC. Nutrição no atleta vegetariano [dissertação]. Porto: Universidade Fernando Pessoa; 2014.

4-RIBEIRO LS, et al. Dieta vegetariana: o que falta saber sobre o papel do selénio na proteção contra doenças? Concurso de Mérito acadêmico do Centro Vegetariano, Portugal, pg. 1-2, 2010.

5-SABATÉ J. The contribution of vegetarian diets to health and disease: a paradigm shift? Am J Clin Nutr. 2003;78 Suppl 3:502S-507S.

6-SLYWITCH E. Alimentação sem carne: Um guia prático para montar sua dieta vegetariana com saúde. Segunda edição. Alaúde, p. 141, 2015.

7-SLYWITCH E. Guia alimentar de dietas vegetarianas para adultos. Sociedade Vegetariana Brasileira; 2012.
8-SVB.ORG. Pesquisa do IBOPE aponta crescimento histórico no número de vegetarianos no Brasil. 2018. Sociedade Vegetariana Brasileira. [acesso 23 jul. 2018] Disponível em: https://www.svb.org.br/2469-pesquisa-doibope-aponta-crescimento-historico-no-numerode-vegetarianos-no-brasil

9-VITOLO M. Nutrição da gestação ao envelhecimento. Rubio: Rio de Janeiro; 2015.

Data de submissão: 01/12/2019

Data de aprovação: 13/12/2019 